\title{
Soft-mode-driven magnetic ordering in the singlet ground-state system PrNi
}

\author{
E.S. Clementyev ${ }^{1,2, *}$, P.A. Alekseev ${ }^{2}$, P. Allenspach ${ }^{1}$, V.N. Lazukov ${ }^{2}$ \\ ${ }^{1}$ Laboratory for Neutron Scattering, ETH Zürich \& Paul Scherrer Institut, 5232 Villigen PSI, Switzerland \\ ${ }^{2}$ Russian Research Centre 'Kurchatov Institute', 123182 Moscow, Russia \\ Received: 16 July 2001/Accepted: 8 January 2002 - @ Springer-Verlag 2002
}

\begin{abstract}
Measurements of the dispersion of the low-energy magnetic modes have been performed by the inelastic neutron scattering technique for the singlet ground state system $\mathrm{PrNi}$ above the Curie temperature $\left(T_{\mathrm{c}}=20.5 \mathrm{~K}\right)$. The neutron spectra were collected in the energy-transfer range up to $7 \mathrm{meV}$. Two well-defined excitons were observed in PrNi along the crystallographic directions [100] and [001]. A progressive softening of the magnetic mode along the [100] direction in the paramagnetic phase at temperatures approaching $T_{\mathrm{c}}$ has been observed at the magnetic Bragg point (zone center) in PrNi. The temperature dependence and the character of the dispersion of the magnetic excitations in $\mathrm{PrNi}$ are indicative of the soft-mode-driven mechanism of the magnetic ordering in this compound.
\end{abstract}

PACS: $71.70 . \mathrm{Ch} ; 71.70 . \mathrm{Gm} ; 78.70 . \mathrm{Nx}$

The magnetic ordering mechanism in singlet ground state systems fundamentally differs from the one in conventional magnets (see [1] and references therein). If the exchange interaction exceeds the critical value with respect to the energy scale of the crystal field (CF) interaction, magnetic ordering occurs by way of the so-called induced magnetic ordering scenario.

A long time ago it was proposed that magnetic ordering in such a system is caused by a soft magnetic mode, which drops in energy at the magnetic Bragg point (zone center for a ferromagnet) [2]. The CF transitions in the paramagnetic regime propagate through the crystal due to the exchange coupling between the magnetic ions; these dispersive magnetic modes are often called CF excitons. However, no significant softening has been observed experimentally in singlet ground state ferromagnets. For example, inelastic neutron scattering (INS) studies of $\mathrm{Pr}_{3} \mathrm{Tl}$ demonstrated almost temperature-independent magnetic excitation spectra in the paramagnetic phase $[3,4]$. We must admit that experimental

*Corresponding author.

(Fax: +49-89/2891-4713, E-mail: eclement@ph.tum.de)

Present address: Physik-Department E21, Technische Universität München, 85748 Germany information on the dispersion of magnetic excitons in singlet ground state systems is rather scarce.

In this paper we report an INS study of the low-energy magnetic modes in PrNi in the paramagnetic domain. The experiments reveal a dramatic softening of the $\mathrm{CF}$ exciton at the zone center in PrNi just above the Curie temperature.

The intermetallic compound PrNi is a ferromagnet with a Curie temperature $T_{\mathrm{c}}$ of about $20 \mathrm{~K}$ [5]. This system crystallizes in the orthorhombic CrB-type structure (space group $\mathrm{Cmcm}$ ). According to bulk measurements on a single crystal, the easy magnetization direction in PrNi is the crystallographic $\boldsymbol{c}$ axis [5]. The Ni contribution to magnetism is negligible in PrNi. The local symmetry in the Pr site is also orthorhombic (point group $\mathrm{C}_{2 v}$ ). The $\mathrm{CF}$ interaction splits the ground $4 f$ multiplet of $\mathrm{Pr}^{3+}$ ions into nine singlets. Therefore the ground state of the $\operatorname{Pr}^{3+} 4 f$ multiplet is necessarily a singlet. Thus PrNi belongs to the family of singlet ground state ferromagnets.

\section{Experiment}

The single crystal of PrNi used in the present work had a volume of about $2 \mathrm{~cm}^{3}$. INS experiments were performed on the triple-axis spectrometers Drüchal and TASP located at the neutron source SINQ (PSI, Villigen). Constant- $Q$ scans were measured at temperatures $21.5 \mathrm{~K}$ to $70 \mathrm{~K}$ for fixed final energies $E_{f}=5.57$ and $8.05 \mathrm{meV}$ (final momentum $k_{f}=1.64$ and $1.97 \AA^{-1}$, respectively). A pyrolytic graphite PG(002) monochromator and analyzer were used. A PG filter was placed after the sample to suppress higher-order contaminations. The measurements were performed in the $\boldsymbol{a}-\boldsymbol{c}$ ([100]/[001]) scattering plane. The INS experiments were done as a function of the reduced wave vector $\boldsymbol{q}$ in pure longitudinal configuration, for $\boldsymbol{q}$ and the total neutron momentum transfer $\boldsymbol{Q}$ along [100] and [001].

\section{Results and discussion}

First of all, the temperature dependence of the intensity at the magnetic Bragg point $\boldsymbol{Q}=(2,0,0)$ has been studied in the 


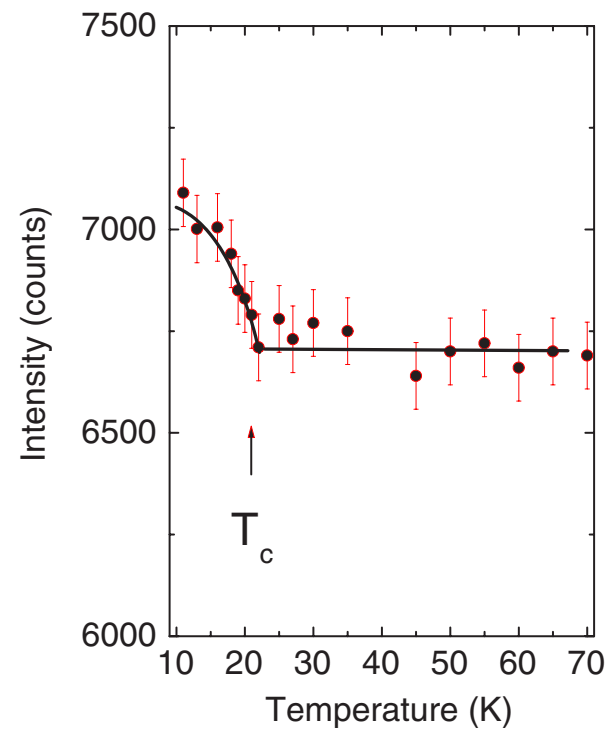

Fig. 1. The temperature dependence of the scattering intensity for PrNi at $\boldsymbol{Q}=(2,0,0)$ obtained on the triple-axis spectrometer Drüchal. The Curie temperature of PrNi is indicated by the arrow. The solid line is a guide for the eye

temperature range $11 \mathrm{~K}$ to $70 \mathrm{~K}$. According to the experimental data shown in Fig. 1, magnetic ordering occurs at about $T=21 \mathrm{~K}$, in good correspondence with the results of macroscopic measurements.

Two well-defined magnetic excitons were observed in the INS spectra of PrNi in the paramagnetic domain. One exciton propagates along the direction [100], another along [001]. Typical neutron spectra collected along these directions are plotted in Figs. 2 and 3.

Both excitons are dispersive magnetic modes. The measurements of the mode along [100] were performed at $T=$ $21.5 \mathrm{~K}, 40 \mathrm{~K}$ and $70 \mathrm{~K}$. The dispersion along the [001] direction was studied at $T=25 \mathrm{~K}, 40 \mathrm{~K}$ and $70 \mathrm{~K}$. A huge

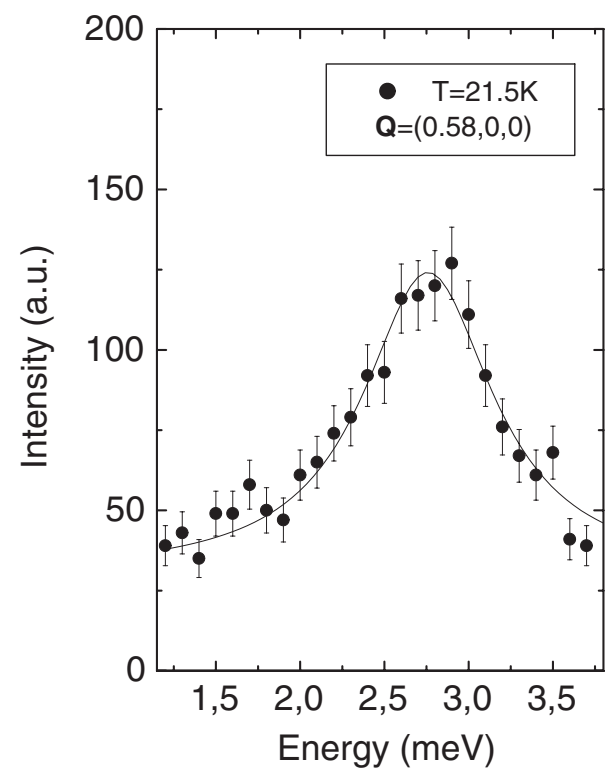

Fig. 2. The INS spectrum of PrNi measured along the [100] crystallographic direction at $\boldsymbol{Q}=(0.58,0,0)$. Neutron data were collected at $T=21.5 \mathrm{~K}$ and fixed final energy $E_{f}=5.57 \mathrm{meV}$. The solid line is a fit to the data

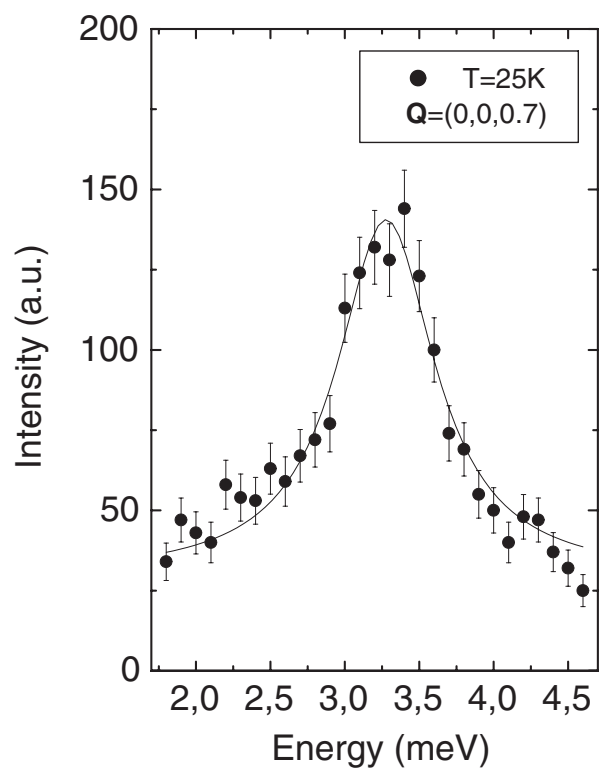

Fig. 3. The neutron spectrum of PrNi measured along the [001] crystallographic direction at $\boldsymbol{Q}=(0,0,0.7)$. The solid line is a fit to the data

Bragg contamination at $\boldsymbol{Q}=(2,0,0)$ did not allow us to measure the magnetic exciton at low temperatures close to $T_{\mathrm{c}}$ since its energy was very close to the elastic peak position. At $T=70 \mathrm{~K}$ the dispersion of the magnetic excitations is relatively weak; at lower temperatures, close to $T_{\mathrm{c}}$, the dispersion is much stronger. Figures 4 and 5 depict the temperature dependences of the dispersion of both magnetic modes in PrNi.

One should note that, due to the symmetry of the reciprocal lattice in $\operatorname{PrNi}$, the point $\boldsymbol{Q}=(2,0,0)$ corresponds to the zone center while $\boldsymbol{Q}=(1,0,0)$ lies on the zone boundary. As to the [001] direction, $\boldsymbol{Q}=(0,0,1)$ is the zone center and $\boldsymbol{Q}=(0,0,0.5)$ is the zone boundary.

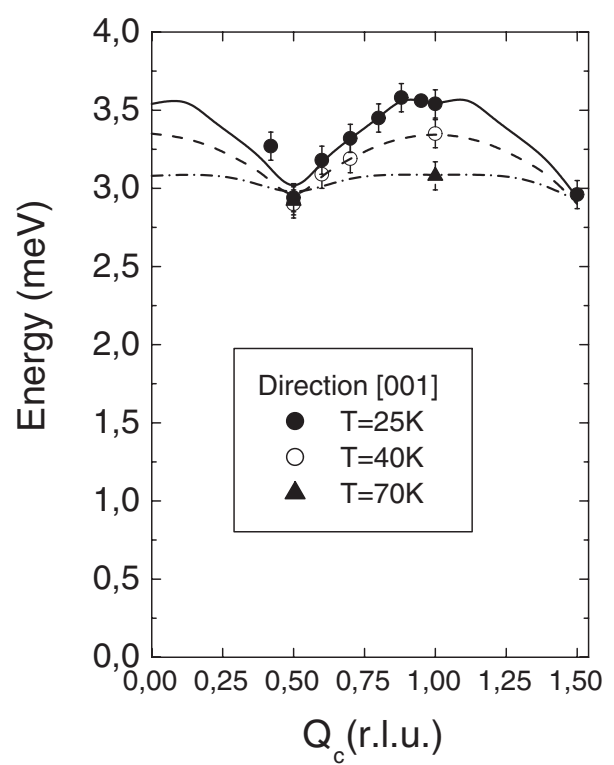

Fig. 4. The dispersion of the magnetic mode in PrNi along the [001] direction at $T=25 \mathrm{~K}$ (filled circles), $40 \mathrm{~K}$ (open circles) and $70 \mathrm{~K}$ (triangles). Lines are guides for the eye 


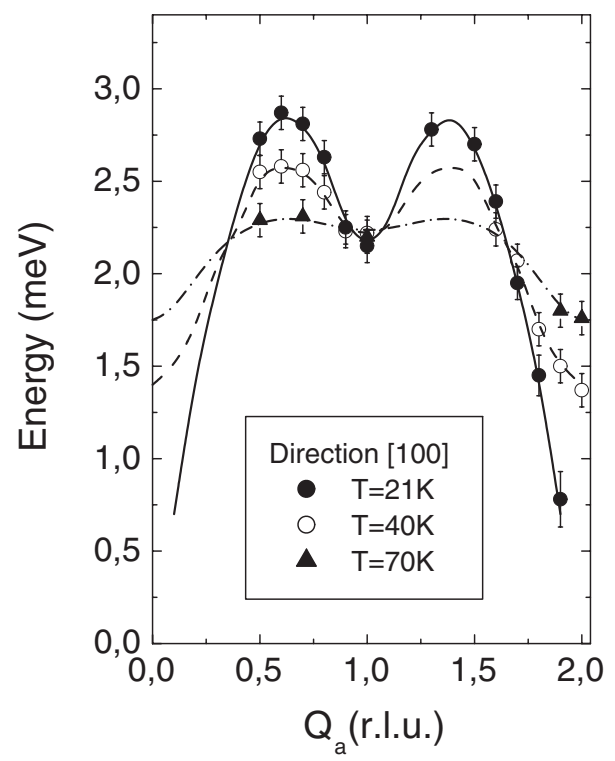

Fig. 5. The dispersion of the magnetic mode in PrNi along the [100] direction at $T=21.5 \mathrm{~K}$ (filled circles), $40 \mathrm{~K}$ (open circles) and $70 \mathrm{~K}$ (triangles). Lines are guides for the eye

The character of the $\boldsymbol{q}$ dependences of the excitations is quite different for the [100] and [001] directions. For $\boldsymbol{q}$ along the crystallographic $c$ axis, the highest exciton energy was observed at the zone center. Along the $a$ axis the magnetic excitation energy has its minimum at the zone center.

The most salient feature of the present study is a strong softening of the magnetic exciton along the [100] direction (see Fig. 5). The softening takes place at the zone center $\boldsymbol{Q}=(2,0,0)$, which becomes a magnetic Bragg point of PrNi below $T_{\mathrm{c}}$. Such an effect can be understood within the random-phase approximation (RPA) model for singlet ground state systems $[1,2]$. The model predicts that the phase of the CF exciton falls to zero. However, the question about the energy of the mode at $T_{\mathrm{c}}$ exactly at $\boldsymbol{Q}=(2,0,0)$ in PrNi remains open due to the Bragg contamination.

Within the simplest RPA-based formalism the CF states are considered as singlets. That is why PrNi (all the CF states are singlets) is close to an ideal model system. Detailed theoretical treatment of the dispersion of the magnetic modes in PrNi requires information on the $\mathrm{CF}$ states in PrNi and on the values of the corresponding matrix elements between the $\mathrm{CF}$ levels.

\section{Summary}

The present study provides an example of the dramatic softening of one of the magnetic modes in a singlet ground state ferromagnetic system. An interplay of the exchange coupling and the CF interaction leads to the dispersive excitons in the paramagnetic phase in PrNi. This compound seems very promising for a detailed study of the soft-mode-driven phase transition in a ferromagnet. Studies of the CF potential and detailed measurements of the dispersion of the magnetic excitations in all high-symmetry directions in $\mathrm{PrNi}$ are now in progress.

\section{References}

1. B.R. Cooper: in Magnetic Properties of Rare Earth Metals, ed. by R.J. Elliott (Plenum, New York 1972)

2. Y.L. Wang, B.R. Cooper: Phys. Rev. 172, 539 (1968)

3. R.J. Birgeneau, J. Als-Nielsen, E. Bucher: Phys. Rev. Lett. 27, 1530 (1971)

4. R.J. Birgeneau, J. Als-Nielsen, E. Bucher: Phys. Rev. B 6, 2724 (1972)

5. G. Fillion, D. Gignoux, F. Givord, R. Lemaire: J. Magn. Magn. Mater. 44, 173 (1984) 